\title{
Association of the COMT vall58met Variant with Antidepressant Treatment Response in Major Depression
}

\author{
Bernhard T Baune*,',2, Christa Hohoff', Klaus Berger ${ }^{3}$, Anna Neumann', Sünke Mortensen', \\ Tilmann Roehrs', Jürgen Deckert', Volker Arolt' and Katharina Domschke' \\ 'Department of Psychiatry, University of Muenster, Muenster, Germany; ${ }^{2}$ Department of Psychiatry, James Cook University, Townsville, QLD, \\ Australia; ${ }^{3}$ Institute of Epidemiology and Social Medicine, University of Muenster, Muenster, Germany; ${ }^{4}$ Department of Psychiatry, University \\ of Wuerzburg, Wuerzburg, Germany
}

\begin{abstract}
In several previous biochemical, pharmacological, and genetic studies, the catechol-O-methyltransferase (COMT) has been suggested to be involved in the pathogenesis as well as the pharmacological treatment of affective disorders. In the present study, 256 patients with major depression (DSM-IV) of Caucasian descent were genotyped for the functional COMT val I 58met polymorphism and characterized for clinical response to antidepressive pharmacological treatment as measured by intra-individual changes of Hamilton Depression (HAM-D-2I) scores over 6 weeks. The COMT I 58val/val genotype conferred a significant risk of worse response after 4-6 weeks of antidepressant treatment in patients with major depression (week 4: $p=0.003$; week 5: $p<0.000$ I; week 6: $p<0.000$ I) after Bonferroni correction for multiple comparisons. The present results strongly point toward a negative influence of the higher activity COMT I58val/ val genotype on antidepressant treatment response during the first 6 weeks of pharmacological treatment in major depression, possibly conferred by consecutively decreased dopamine availability. This finding suggests a potentially beneficial effect of an antidepressive addon therapy with substances increasing dopamine availability individually tailored according to COMT vall 58 met genotype. Neuropsychopharmacology (2008) 33, 924-932; doi:I0.I 038/sj.npp. I 30I462; published online 23 May 2007
\end{abstract}

Keywords: major depression; catechol-O-methyltransferase; COMT vall 58met; polymorphism; antidepressants; treatment response

\section{INTRODUCTION}

Genetic factors have been suggested to play a pivotal role in the individual response to antidepressant treatment. Pharmacogenetic studies aim at identification of genetic variations that influence drug targets or factors interfering with drug action. A number of pharmacogenetic studies have examined association of response to antidepressants with variation in candidate genes, particularly those involved in the serotonergic system (eg, serotonin transporter (5-HTT), serotonin receptor $2 \mathrm{~A}(5-\mathrm{HT} 2 \mathrm{~A})$, monoamine oxidase A, tryptophan hydroxylase, for review see Serretti et al, 2005). Only few studies have focused on candidate genes implicated in the noradrenergic or dopaminergic systems, which, however, have been shown to have a significant impact on the pathogenesis of depression and also to interact with serotonergic pathways in the pharmacological treatment of the disease. In line with the monoamine hypothesis of depression, a deficit in brain norepinephrine

*Correspondence: Professor BT Baune, Department of Psychiatry, School of Medicine, James Cook University, Townsville, QLD 48II, Australia, Tel: +61747816731, Fax: +61747816219,

E-mail: bernhard.baune@jcu.edu.au

Received 21 December 2006; revised 26 March 2007; accepted 25 April 2007 and dopamine has been demonstrated in patients with major depression, which was reversible following pharmacological blockade of the norepinephrine transporter with desipramine. However, the patients' clinical improvement was most significantly correlated with dopamine turnover (Lambert et al, 2000). Accordingly, in the Flinders sensitive line rat model of depression, decreased availability of extracellular dopamine in the nucleus accumbens was found to be reversible by treatment with serotonergic antidepressants accompanied by improvements in depressive-like behavior (Zangen et al, 2001; Dremencov et al, 2004). Reciprocally, blockade of dopamine D2/D3 receptors has been reported to acutely reverse the antidepressant effect of selective serotonin reuptake inhibitors (SSRIs) in animal models of depression as well as in patients suffering from major depression (Willner, 2002; Willner et al, 2005). This is in line with the clinical finding that augmentation of antidepressant treatment with sustained-release bupropion, an inhibitor of dopamine reuptake, as well as the dopamine agonists pramipexole and ropinirole resulted in significant reduction in the number and severity of depressive symptoms after failure of therapy with SSRIs (Trivedi et al, 2006; Cassano et al, 2004, 2005). Additionally, pramipexole has been shown to ameliorate depressive symptoms in addition to its effects on motor symptoms in Parkinson's disease (Barone et al, 2006). 
The catechol-O-methyltransferase (COMT) plays a pivotal role in the degradation of norepinephrine and dopamine (Mannisto and Kaakkola, 1999) and therefore serves as a promising candidate in pharmacogenetic studies of antidepressant action. In patients with major depression, significantly elevated erythrocyte COMT activity was reported (Shulman et al, 1978; Davidson et al, 1979). Accordingly, tolcapone, a COMT inhibitor, has been shown to reverse an anhedonic state in a rat model of depression, suggesting a potential role of tolcapone in the treatment of depression in addition to its efficacy in Parkinson's disease (Moreau et al, 1994). In a clinical pilot study on the efficacy of tolcapone in the treatment of major depressive disorder, a significant reduction in depression severity was observed (Fava et al, 1999).

The COMT gene (NM_000754) maps to chromosome 22q11.2, and contains a functional 472G/A single-nucleotide polymorphism (rs4680) causing an amino-acid substitution from valine to methionine in codon 158 (val158met) of the membrane-bound form of the enzyme (codon 108 (vallo8met) of the soluble form). The valine allele (472G) has initially been reported to result in a three- to fourfold higher COMT activity as compared to the methionine allele (472A) (Lachman et al, 1996). However, the valine allele appears to be less expressed in the brain as compared to the methionine allele (Bray et al, 2003; Zhu et al, 2004), which slightly mitigates the effect to about $40 \%$ increased enzyme activity as conferred by the valine allele (Chen et al, 2004). The COMT val158met polymorphism has repeatedly been investigated for association with major depression with contradictory reports of no association (Kunugi et al, 1997; Frisch et al, 1999; Cusin et al, 2002; Serretti et al, 2003), possible association with the valine allele (Massat et al, 2005; Funke et al, 2005) or conversely the methionine allele (Ohara et al, 1998). Two published studies on the role of the COMT val158met polymorphism in antidepressant treatment response investigating samples of 102 and 346 patients, respectively, report a tentative negative effect of the COMT $158 \mathrm{met} / \mathrm{met}$ genotype on mirtazapine and citalopram response in major depression (Szegedi et al, 2005; Arias et al, 2006).

In the present study, the influence of the COMT val158met polymorphism on clinical response to antidepressants was investigated in samples of Caucasian patients with major depression and bipolar disorder, major depressive episode, by means of a novel approach analyzing the intra-individual rather than the averaged course of therapy response, in an attempt to follow the recently published American College of Neuropsychopharmacology (ACNP) task force guidelines on response and remission in major depressive disorder (Rush et al, 2006) and therefore to possibly further clarify the role of COMT gene variation in antidepressant treatment response. Since biochemical and pharmacological studies provide support for decreased availability of dopamine and norepinephrine as a detrimental factor in the pathomechanism of depression as well as response to pharmacological antidepressive treatment, higher COMT activity, as conferred by the COMT 158val allele leading to decreased dopamine and norepinephrine availability, was hypothesized to have a negative effect on antidepressant drug response in depression. In addition, given the inconsistent data on COMT val158met impact on major depression published so far, we aimed at further elucidation of the influence of the COMT val158met polymorphism on the pathogenesis of major depression using a categorical association study design.

\section{MATERIALS AND METHODS}

\section{Samples}

Samples of 268 unrelated Caucasian patients with a current major depressive episode (mean age: $49.7 \pm 15.4$ years; $\mathrm{f}=154, \mathrm{~m}=114)$ and 72 patients with bipolar disorder, major depressive episode, admitted for inpatient treatment were consecutively recruited at the Department of Psychiatry, University of Muenster, Germany, between 2004 and 2006. For pharmacogenetic analyses, only patients with an HAM-D admission score $>10$ and a treatment cycle of at least 6 weeks from baseline were considered, leaving a sample of $N=256$ patients with major depression (mean age: $50.4 \pm 14.9$ years; $\mathrm{f}=145, \mathrm{~m}=111$ ) and 65 patients with bipolar disorder, major depressive episode (mean age: $45.9 \pm 14.5$ years; $f=39, \mathrm{~m}=26$ ). Patients with schizoaffective disorders or comorbid substance abuse disorders, mental retardation, neurological or neurodegenerative disorders, impairing psychiatric evaluation were not included in this analysis. In order to minimize the risk of ethnic stratification, Caucasian descent was ascertained by Caucasian background of both parents.

The control group consisted of 557 (mean age: $50.2 \pm 14.2$ years; $f=302, m=255$ ) age- and gender-matched healthy subjects of German descent representative of the general population, where the presence of clinically relevant depressive symptoms was excluded using the Centre for Epidemiological Studies Depression scale (CES-D) (Radloff, 1977).

The ethics committee of the University of Muenster (Muenster, Germany) approved the study. After complete description of the study to the subjects, written informed consent was obtained.

\section{Assessment}

Patients' diagnoses were obtained by the use of a structured clinical interview (SCID-I) according to the criteria of DSMIV (Wittchen et al, 1997), and all patients were characterized for family history of psychiatric disorders in firstdegree relatives. Clinical course of depression was assessed with the Hamilton Depression (HAM-D-21) scale, the Beck's Depression Inventory (BDI), the Clinical Global Impression (CGI) scale, and the Global Assessment of Functioning (GAF) scale. While scores on the HAM-D-21 were obtained on a weekly basis, BDI, CGI, and GAF scores were obtained at admission and discharge only. Side effects were not systematically assessed in detail; however, there were no dropouts due to side effects. Age- and gender-matched healthy controls were assessed for depressive symptoms using the CES-D scale.

\section{Response}

Clinical response to treatment was measured by the intraindividual changes of HAM-D-21 scores over the 6 weeks 
study period. This is in accordance with the recently published ACNP task force guidelines on response and remission in major depressive disorder, which recommend monitoring of changes within a subject (for whom initial severity is fixed) rather than comparing response rates between subjects for whom initial values range widely (Rush et al, 2006). The intra-individual weekly change score introduced here considered the weekly changes in HAM-D scores after week 1 up to week 6 . The initial changes in HAM-D occurring during week 1 were not included, as HAM-D changes during this period were most likely not related to antidepressant effects since antidepressants have been regularly switched in this cohort of inpatients during week 1. Thus, the intra-individual weekly change on the HAM-D scale per subject was captured by calculating the raw HAM-D score at weeks 2-6 minus HAM-D at the end of week 1. Negative numbers of the change score (based on raw scores) indicate a reduction on the HAM-D score (larger numbers indicate better progress than lower numbers) over time, whereas positive scores indicate increase of HAM-D scores and a deterioration of mental state over time, respectively. In additional analyses (Figure 2), a weekly percent change scores was obtained as percent change relative to week 1 . This model allows for direct comparison of intra-individual response dynamics over time. In addition, it corrects for improvements unrelated to antidepressant action occurring in the first week after admission due to the admission itself or concomitant benzodiazepine and antipsychotic medication with rapid onset of action.

\section{Medication}

Patients were treated in a naturalistic setting with a variety of antidepressant medication (mirtazapine: $N=28$ (10.9\%), citalopram/escitalopram: $N=38$ (14.8\%), venlafaxine: $N=45(17.6 \%)$, mirtazapine plus citalopram/escitalopram: $N=38$ (14.8\%), mirtazapine plus venlafaxine: $N=63$ $(n=24.6 \%)$, other (TCA, MAO inhibitors, lithium): $N=44$ $(17.2 \%)$ ). As co-medication atypical neuroleptics (quetiapine, olanzapine, risperidone: $N=121,47.3 \%$ ) as well as mood stabilizers (lithium, valproate acid: $N=60,23.4 \%$ ) were used in addition to antidepressant treatment. Benzodiazepines were used in three cases only.

\section{Genotyping}

According to published protocols (Domschke et al, 2004), fragments containing the COMT val158met polymorphism were amplified with primers COMT-F (5'-TCACCATC GAGATCAACCCC) and COMT-R (5'-ACAACGGGTCAGG CATGCA). Standard PCR was carried out in a $20 \mu \mathrm{l}$ volume containing $60 \mathrm{ng}$ of genomic DNA, $10 \mathrm{pmol}$ of each primer, $200 \mu \mathrm{M}$ dNTPs, $0.4 \mathrm{U} \mathrm{Taq}^{\mathrm{TM}}$ DNA polymerase (Eppendorf AG, Hamburg, Germany), $50 \mathrm{mM} \mathrm{KCl,} 2.5 \mathrm{mM} \mathrm{MgCl}_{2}$, and $10 \mathrm{mM}$ Tris- $\mathrm{HCl}(\mathrm{pH} \mathrm{8.4})$. After an initial $5 \mathrm{~min}$ denaturation at $94^{\circ} \mathrm{C}, 35$ cycles were carried out consisting of $30 \mathrm{~s}$ at $94^{\circ} \mathrm{C}, 30 \mathrm{~s}$ at the annealing temperature of $64^{\circ} \mathrm{C}$, and $60 \mathrm{~s}$ at $72^{\circ} \mathrm{C}$, followed by a final extension time of $10 \mathrm{~min}$ at $72^{\circ} \mathrm{C}$ in a T-Gradient PCR System (Biometra, Goettingen, Germany). Genotyping was performed by means of a restriction fragment length polymorphism (RFLP) assay with the restriction enzyme NlaIII ( $3 \mathrm{U}$ ), as recommended by the manufacturer (New England Biolabs, Frankfurt, Germany), resulting in 64,18 , and $13 \mathrm{bp}$ bands for the A-allele (158met) and 82 and $13 \mathrm{bp}$ bands for the G-allele (158val), respectively. A $6.7 \mu \mathrm{l}$ portion of the digested product was mixed with $10 \mu \mathrm{l}$ denaturing solution and separated for $2 \mathrm{~h}$ on a $15 \%$ polyacrylamide gel (acrylamide : bisacrylamide $=37.5: 1$; Multigel-Long/Biometra, Goettingen, Germany) containing $1 \times \mathrm{TBE}$ at $230 \mathrm{~V} / \mathrm{cm}$. Bands were visualized by silver-staining. To minimize the risk of genotyping errors, 60 randomized patients and 120 healthy controls were additionally genotyped by TaqMan $5^{\prime}$-exonuclease assays using different colored fluorophores for allele labeling and allelic discrimination by fluorescence signal detection (Applied Biosystems, Darmstadt, Germany), which yielded a $100 \%$ congruence rate with results obtained using the RFLP assay. Preparation of PCR reaction mixtures was performed by Genesis Workstation RSP 150 (Tecan, Crailsheim, Germany). For PCR amplification and allelic discrimination, the ABI Prism 7900HT Sequence Detection System and SDS software version 2.1 (Applied Biosystems) were used. These combined genotyping methods yielded a genotyping completion rate of $100 \%$ for all included patients and controls. Genotypes were determined by investigators blinded for clinical diagnoses.

\section{Statistical Analysis}

The categorical association analysis of allele and genotype distribution across patients with major depression $(N=268)$ and age- and gender-matched healthy controls $(N=557)$ was performed by means of Armitage's trend test, as provided by the program DeFinetti available as an online source (http://ihg.gsf.de/cgi-bin/hw/hwal.pl; Sasieni, 1997).

Comparisons of HAMD, BDI, CGI, and GAF baseline scores across genotype groups were carried out with oneway ANOVA ( $>2$ categories) (Table 1). The pharmacogenetic investigation of COMT val158met genotype effects on HAM-D change scores over 6 weeks of antidepressant treatment was performed using an overall ANOVA with repeated measures (genotype as fixed factor, and time point as repeated measure; adjusted for age, gender, and family history of psychiatric disorders) with post hoc Bonferroni correction for multiple comparisons (Table 2).

In addition to the overall ANOVA with repeated measures, we performed separate multiple ANOVAs with post hoc Bonferroni test (adjusted for age, gender, and family history of psychiatric disorders) to obtain results on the weekly intra-individual HAM-D change scores across genotype groups. Bonferroni correction for multiple comparisons (15 tests per independent variable in Table 3 ) set the limit of the $p$-value to $p \leqslant 0.003$. In addition, stratified analyses for gender and family history of mental disorders were performed as shown in Tables 2 and 3.

With these parameters, for continuous measurements, our sample had a high power (80\%) to detect a difference of at least 2.4 points on the HAM-D scale between two genotypes. Hardy-Weinberg equilibrium was examined using the program Finetti provided as an online source (http://ihg.gsf.de/cgi-bin/hw/hwa1.pl; Sasieni, 1997). 
Table I COMT vall58met Genotype Frequencies and Genotype Comparisons for Clinical Characteristics at Baseline Stratified for Gender and Family History

\begin{tabular}{|c|c|c|c|c|}
\hline \multirow{2}{*}{$\begin{array}{l}N=256 \\
\text { COMT VI58M genotype }\end{array}$} & \multicolumn{3}{|c|}{ Patients with major depression } & \multirow[b]{2}{*}{$p$-value } \\
\hline & $\mathrm{val} / \mathrm{val}$ & $\mathrm{val} / \mathrm{met}$ & met/met & \\
\hline Total (n) & 54 & 133 & 69 & \\
\hline HAMD baseline (mean) & 22.9 & 23.4 & 22.8 & $>0.05$ \\
\hline BDI baseline (mean) & 26.6 & 27.5 & 28.1 & $>0.05$ \\
\hline GAF baseline (mean) & 43.7 & 42.1 & 44.1 & $>0.05$ \\
\hline CGI baseline (mean) & 5.5 & 5.6 & 5.4 & $>0.05$ \\
\hline Males (n) & 27 & 51 & 33 & \\
\hline HAMD baseline (mean) & 21.7 & 24.4 & 21.3 & $>0.05$ \\
\hline BDI baseline (mean) & 24.6 & 29.3 & 26.9 & $>0.05$ \\
\hline GAF baseline (mean) & 44.1 & 44.5 & 42.8 & $>0.05$ \\
\hline CGI baseline (mean) & 5.4 & 5.7 & 5.3 & $>0.05$ \\
\hline Females (n) & 27 & 82 & 36 & \\
\hline HAMD baseline (mean) & 24.2 & 22.7 & 24.2 & $>0.05$ \\
\hline BDI baseline (mean) & 28.5 & 26.4 & 29.2 & $>0.05$ \\
\hline GAF baseline (mean) & 43.4 & 42.3 & 43.7 & $>0.05$ \\
\hline CGI baseline (mean) & 5.5 & 5.5 & 5.6 & $>0.05$ \\
\hline Family history positive (n) & 20 & 57 & 35 & \\
\hline HAMD baseline (mean) & 20.3 & 24.2 & 22.5 & $>0.05$ \\
\hline BDI baseline (mean) & 25.4 & 27.6 & 27.0 & $>0.05$ \\
\hline GAF baseline (mean) & 47.0 & 42.5 & 44.2 & $>0.05$ \\
\hline CGI baseline (mean) & 5.3 & 5.7 & 5.3 & $>0.05$ \\
\hline Family history negative ( $n$ ) & 34 & 76 & 34 & \\
\hline HAMD baseline (mean) & 24.5 & 22.7 & 23.2 & $>0.05$ \\
\hline BDI baseline (mean) & 27.3 & 27.5 & 29.3 & $>0.05$ \\
\hline GAF baseline (mean) & 42.0 & 41.8 & 43.9 & $>0.05$ \\
\hline CGI baseline (mean) & 5.6 & 5.5 & 5.6 & $>0.05$ \\
\hline
\end{tabular}

p-value of comparisons between genotypes by one-way ANOVA.

\section{RESULTS}

In our sample of 268 patients and an age- and gendermatched control sample of 557 healthy probands, no association of the COMT val158met polymorphism with major depression was observed (patients: val/val: 55, val/ met: 142 , met/met: 71 ; controls: val/val: 112 , val/met: 275 , met/met: 170; common odds ratio $=1.091, \chi^{2}=0.73$, $p=0.3916)$. Hardy-Weinberg equilibrium was fulfilled for the case $(p=0.30)$ as well as the control sample $(p=0.97)$.

The pharmacogenetic sample was comprised of 256 patients with major depression (mean age: $50.4 \pm 14.9$; $\mathrm{f}=145, \mathrm{~m}=111)$. The subsamples stratified for gender and family history of mental diseases did not significantly differ for age, education, marital status, or comorbidity with anxiety disorders. COMT val158met genotype frequencies in the overall pharmacogenetic sample of patients with major depression as well as the respective subgroups stratified for gender and family history of mental diseases are given in Table 1. The distribution of COMT val158met genotypes did not significantly differ from the expected numbers calculated on the basis of observed allele frequencies according to the Hardy-Weinberg equilibrium $(p=0.53)$.

The mean HAM-D score at admission was $23.1 \pm 7.3$ and at discharge was $6.0 \pm 5.0$, without showing any differences between gender and family history of mental disorders. Table 1 shows no significant differences of HAM-D, BDI, GAF, and CGI baseline scores between COMT val158met genotypes in the overall sample as well as stratified for gender or family history of mental illness in first-degree relatives.

The raw HAM-D scores over the course of 6 weeks are presented in Figure 1, indicating only a marginal change over time for patients with the COMT158val/val genotype (mean change of $-1.6 \pm 0.8 ; p=0.13$ ). Moreover, while HAM-D scores significantly decreased over 6 weeks in patients with either the val/met (mean change of $-5.6+0.7$; $p<0.05$ ) or met/met (mean change of $-4.5 \pm 1.0 ; p<0.05$ ) genotypes, after an initial slight decline, patients with the $\mathrm{val} / \mathrm{val}$ genotype showed no major change in HAM-D scores after 3 weeks of antidepressant treatment.

Table 2 presents results of the overall ANOVA with repeated measures (genotype as fixed factor, and time point as repeated measure; post hoc Bonferroni correction) for the HAM-D change scores over 6 weeks (dependent variable) of antidepressant treatment across genotype groups stratified for gender and family history. The COMT 158val/val genotype as compared to the heterozygous $158 \mathrm{val} / \mathrm{met}$ genotype conferred a significant increased risk of worse response to antidepressant treatment over 6 weeks. In analyses stratified for gender and family history, the COMT $158 \mathrm{val} / \mathrm{val}$ genotype as compared to the heterozygous $158 \mathrm{val} / \mathrm{met}$ genotype showed an increased risk for worse antidepressant treatment response after 6 weeks in men $(p<0.049)$, women $(p<0.005)$, and subjects with a negative $(p<0.005)$ or positive $(p<0.05)$ family history for psychiatric disorders in first-degree relatives (Table 2). Figure 2 demonstrates the HAM-D percent changes relative to week 1 across genotypes. While patients with the val/val genotype hardly showed any changes on the HAM-D score over time, the other two genotypes were related to a reduction in HAM-D scores with pronounced effects for the val/met genotype (Figure 2).

In Table 3, separate multivariable ANOVA analyses of the HAM-D weekly change between weeks 1 and 6 (based on raw scores) were performed across genotype groups, stratified for gender and family history, and results were adjusted for Bonferroni correction for multiple comparisons (significance level set to $p \leqslant 0.003$ ). The COMT 158val/ val genotype as compared to the heterozygous $158 \mathrm{val} / \mathrm{met}$ genotype conferred a significant risk of worse response to antidepressant treatment for the overall change score at weeks $4(p<0.003), 5(p<0.0001)$, and $6(p<0.0001)$. In addition, stratified analyses for history of psychiatric diseases in first-degree relatives revealed a significant effect of the COMT $158 \mathrm{val} / \mathrm{val}$ genotype as compared to the heterozygous $158 \mathrm{val} / \mathrm{met}$ genotype in subjects without family history of psychiatric disorders $(p<0.001)$ at week 5 after start of antidepressant treatment (Table 3). Similar 
Table 2 Overall Reduction in Mean HAM-D Scores Across COMT val I 58met Genotypes Stratified for Gender and Family History during a 6-Week Course of Antidepressant Treatment

\begin{tabular}{|c|c|c|c|c|}
\hline \multirow{3}{*}{$\begin{array}{l}N=256 \\
\text { COMT VI58M } \\
\text { genotype }\end{array}$} & \multicolumn{3}{|c|}{ Patients with major depression } & \multirow[b]{3}{*}{$p$-value } \\
\hline & val/val & val/met & met/met & \\
\hline & mean HAM-D reduction & mean HAM-D reduction & mean HAM-D reduction & \\
\hline \multicolumn{5}{|l|}{ Total } \\
\hline HAM-D change score ${ }^{\mathrm{a}, \mathrm{b}}$ & -1.6 & -5.6 & -4.5 & $<0.000 I^{*}$ \\
\hline \multicolumn{5}{|l|}{ Males } \\
\hline HAM-D change scores ${ }^{b, c}$ & -2.3 & -5.9 & -4.7 & $<0.049 *$ \\
\hline \multicolumn{5}{|l|}{ Females } \\
\hline HAM-D change scores ${ }^{b, c}$ & -1.1 & -5.3 & -4.5 & $<0.005^{*}$ \\
\hline \multicolumn{5}{|l|}{ Family history positive } \\
\hline HAM-D change scores ${ }^{b, d}$ & -1.7 & -5.6 & -4.3 & $<0.05^{*}$ \\
\hline \multicolumn{5}{|l|}{ Family history negative } \\
\hline HAM-D change scores ${ }^{\mathrm{b}, \mathrm{d}}$ & -1.7 & -5.6 & -4.6 & $<0.005^{*}$ \\
\hline
\end{tabular}

results were obtained using ANOVA with repeated measures (overall change at weeks 1-6) and separate ANOVAs (weekly change), when a HAM-D percent change relative to week 1 was employed (data not shown).

When investigating subgroups stratified for the type of antidepressant medication, the negative influence of the COMT 158val/val genotype on response was significant for medication with mirtazapine plus venlafaxine or citalopram/escitalopram $(N=101 ; p<0.005)$, as compared to the COMT 158val/met genotype. This effect was most prominent after 4, 5, and 6 weeks of treatment (week 4: $p<0.019$; week 5: $p<0.001$; week 6: $p<0.001$ ). No significant COMT val158met genotype effects on HAM-D treatment response were found among patients receiving single antidepressants (escitalopram, mirtazapine, venlafaxine). Exposure to atypical neuroleptics or mood stabilizers was not different between carriers of the COMT 158val and 158met alleles, and atypical antipsychotics or mood stabilizers were not related to treatment response after 6 weeks.

Significant effects of the COMT val158met genotype on BDI, GAF, and CGI change scores between admission and discharge were not observed (each measure was assessed at baseline and discharge) (data not shown).

In the subsample of patients with bipolar disorder, major depressive episode $(N=65$; val/val: 12 , val/met: 35 , met/ met: 18), no influence of the COMT val158met variant on HAM-D change scores could be detected (mean reduction in HAM-D scores between weeks 1 and 6: val/val: -4.31 ; val/met: -4.87; met/met: -3.91; ANOVA with repeated measures $p=0.786)$. Furthermore, there was no significant difference when comparing genotype frequencies between patients with major depression and patients with bipolar disorder, major depressive episode $\left(\chi^{2}=0.12\right.$; $p=0.729)$.

\section{DISCUSSION}

The present observations do not support a major role of the COMT val158met variant in the pathogenesis of major depression, confirming previous findings (Kunugi et al, 1997; Frisch et al, 1999; Cusin et al, 2002; Serretti et al, 2003).

However, applying an innovative intra-individual assessment approach of response to treatment, our results suggest a strong detrimental effect of the higher activity COMT $158 \mathrm{val} / \mathrm{val}$ genotype on antidepressant treatment response in patients with major depression, but not with bipolar disorder. In major depressive patients homozygous for the COMT 158val allele, increased COMT activity resulting in a decreased availability of dopamine and norepinephrine might impair the pharmacological efficacy of serotonergic and noradrenergic antidepressants during the first 6 weeks of treatment and thus be, in part, responsible for $30-40 \%$ of patients not initially responding to antidepressant medication (Sackeim, 2001).

In the first of two previously published studies on COMT gene influence on antidepressant response, German patients carrying the COMT 158met/met genotype showed a slightly slower and poorer response to treatment with mirtazapin $(N=53)$ (Szegedi et al, 2005). Arias et al (2006) observed a disadvantage for homozygous carriers of the COMT 158met allele in early remission at the $6 \mathrm{th} / 8$ th week of treatment with citalopram in a Spanish sample of 139 patients with 
Table 3 Weekly Reduction of Raw HAM-D Scores Across COMT vall 58met Genotypes Stratified for Gender and Family History

\begin{tabular}{|c|c|c|c|c|}
\hline \multirow{2}{*}{$\begin{array}{l}N=256 \\
\text { COMT VI58M }\end{array}$} & \multicolumn{3}{|c|}{ Patients with major depression } & \multirow{2}{*}{$p$-value } \\
\hline & $\mathrm{val} / \mathrm{val}$ & $\mathrm{val} / \mathrm{met}$ & met/met & \\
\hline \multicolumn{5}{|l|}{ Total } \\
\hline HAMD change score at week $2^{a-d}$ & -0.5 & -3.0 & -2.5 & 0.018 \\
\hline HAMD change score at week $5^{\text {a-d }}$ & -1.8 & -6.9 & -5.6 & $0.0001 * *$ \\
\hline HAMD change score at week $6^{a-d}$ & -1.7 & -8.1 & -6.1 & 0.000 I*** \\
\hline \multicolumn{5}{|l|}{ Males } \\
\hline HAMD change score at week $2^{\mathrm{a}, \mathrm{b}, \mathrm{d}}$ & -0.7 & -2.7 & -2.5 & 0.630 \\
\hline HAMD change score at week $6^{\mathrm{a}, \mathrm{b}, \mathrm{d}}$ & -3.5 & -9.1 & -6.9 & 0.030 \\
\hline \multicolumn{5}{|l|}{ Females } \\
\hline HAMD change score at week $2^{\mathrm{a}, \mathrm{b}, \mathrm{d}}$ & -0.01 & -3.2 & -2.5 & 0.029 \\
\hline HAMD change score at week $3^{\mathrm{a}, \mathrm{b}, \mathrm{d}}$ & -0.8 & -4.0 & -3.3 & 0.085 \\
\hline HAMD change score at week $4^{\mathrm{a}, \mathrm{b}, \mathrm{d}}$ & -1.2 & -5.1 & -4.8 & 0.054 \\
\hline HAMD change score at week $5^{\mathrm{a}, \mathrm{b}, \mathrm{d}}$ & -1.2 & -6.7 & -6.0 & 0.006 \\
\hline HAMD change score at week $6^{a, b, d}$ & -1.8 & -7.4 & -5.6 & 0.020 \\
\hline \multicolumn{5}{|l|}{ Family history positive } \\
\hline \multicolumn{5}{|l|}{ Family history negative } \\
\hline HAMD change score at week $2^{\mathrm{a}-\mathrm{c}}$ & -0.5 & -2.7 & -2.2 & 0.164 \\
\hline HAMD change score at week $3^{a-c}$ & -1.6 & -4.1 & -3.8 & 0.206 \\
\hline HAMD change score at week $4^{\mathrm{a}-\mathrm{c}}$ & -1.5 & -5.9 & -5.7 & 0.013 \\
\hline HAMD change score at week $5^{a-c}$ & -1.5 & -7.3 & -5.7 & $0.001 * * *$ \\
\hline HAMD change score at week $6^{\mathrm{a}-\mathrm{c}}$ & -2.5 & -7.8 & -5.8 & 0.019 \\
\hline
\end{tabular}

${ }^{\mathrm{a}}$ Single multivariable ANOVA for weekly reduction of raw HAM-D change scores.

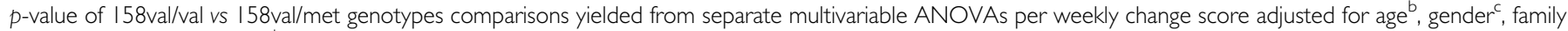
history of psychiatric illness ${ }^{d}$ and with post hoc Bonferroni correction.

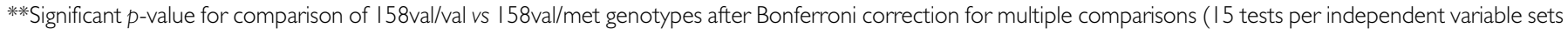
$p$-value to 0.003 ).

major depression. These findings of a negative influence of the $158 \mathrm{met} / \mathrm{met}$ genotype appear to be in contrast with the present observation of the $158 \mathrm{val} / \mathrm{val}$ genotype conferring higher risk of non-response. However, the presently observed 158val/val genotype effect was most prominent when compared to the $158 \mathrm{val} /$ met genotype rather than the $158 \mathrm{met} / \mathrm{met}$ genotype. This might be explained in analogy to the proposed 'inverted $U$ ' relationship between dopamine levels and prefrontal function. Under this model, function has been suggested to be optimal within a narrow range of dopamine activity, with too little or too much dopamine having relatively deleterious effects (Mattay et al, 2003). Thus, both the 158met/met genotype increasing dopamine levels as well as the $158 \mathrm{val} / \mathrm{val}$ genotype impairing dopamine availability might contribute to the risk of nonresponse to antidepressant treatment, which across different studies might be modulated by factors extrinsic to the COMT $158 \mathrm{val} / \mathrm{met}$ locus such as a specific constellation of 


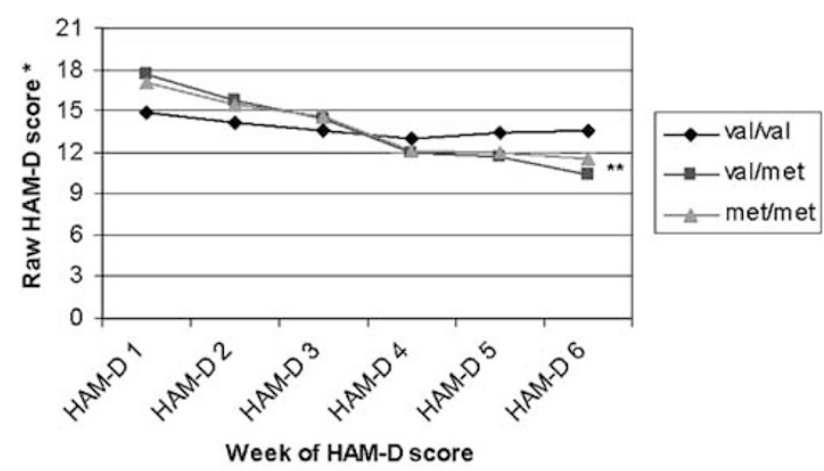

* adjusted for age, gender and family history of psychiatric disorders "* change of HAM-D for both val/met and met/met genotypes between week 1 and 6 was significant at $p<0.05$

Figure I Raw HAM-D scores between weeks I and 6 of antidepressant treatment across COMT vall 58 met genotypes in 256 subjects with major depression.

genetic risk factors within the dopaminergic system, differential environmental risk factors, or different subclinical characteristics related to reduced or increased dopamine tonus in the respective samples of patients with depression (for review see Dailly et al, 2004). This notion of dose-dependency in the dopaminergic tonus with respect to depressive symptoms can be further illustrated by a case report observing improved depressive symptomatology on augmentation of antidepressant medication with bromocriptine $2.5-5 \mathrm{mg} / \mathrm{day}$, whereas an increased dose of bromocriptine $15 \mathrm{mg}$ /day resulted in a deteriorated clinical status (Wada et al, 2001). Additionally, differences in antidepressive medication across studies may account for the discrepant findings with regard to the associated allele.

Interestingly, stratification of patients with major depression for gender revealed that the association of the COMT $158 \mathrm{val} / \mathrm{val}$ genotype with poor outcome after 5-6 weeks of treatment was slightly more pronounced in the female subsample. This observation is in accordance with previous studies in obsessive-compulsive disorder, panic disorder, and schizophrenia reporting association of the COMT val158met polymorphism preferably in female patients (Alsobrook et al, 2002; Domschke et al, 2004; Kremer et al, 2003). Gender-specific effects of COMT gene variation also fit well with findings of sexually dimorphic COMT activity (Boudikova et al, 1990), which might be due to the fact that estrogen can regulate COMT transcription by binding to estrogen response elements in the promoter region of the COMT gene (Xie et al, 1999). Thus, genderspecific effects of the COMT val158met polymorphism might be due to hormonal influences, gene-gene interaction (eg, with a sex-linked gene), or gene-environment interaction with a gender-specific exposure possibly affecting an intermediate phenotype common to several different psychiatric diseases including major depression.

Our study indicates that the use of HAM-D might be more suitable for the detection of differences in psychopathology across COMT val158met genotypes as compared to the subjective measure of the BDI and measures assessing functionality (GAF and CGI), as those measures showed no differences across COMT val158met genotypes. However, this possible interpretation needs to be considered with

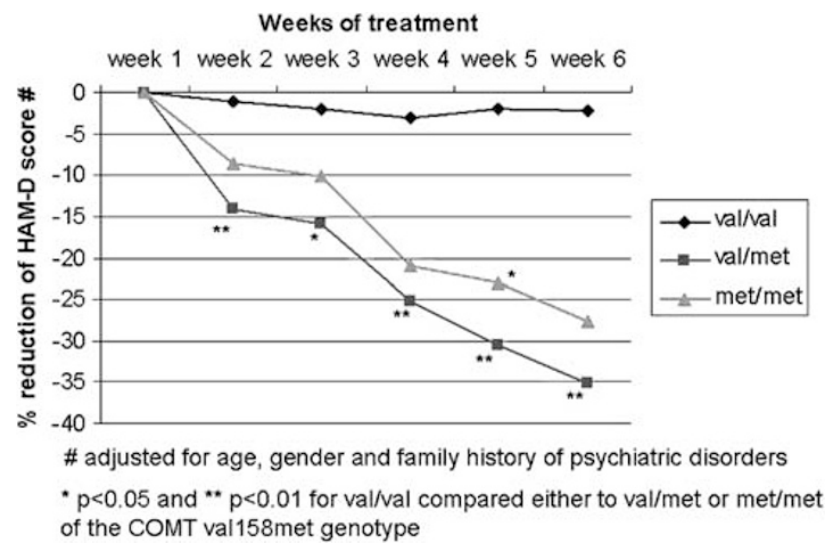

Figure 2 Percent reduction of HAM-D scores over 6 weeks of antidepressant treatment across COMT vall58met genotypes in 256 subjects with major depression.

caution and warrants further clarification as the BDI, GAF, and CGI were only measured at admission and discharge as opposed to the weekly assessment using the HAM-D scale.

The following limitations have to be mentioned and considered while interpreting the present results: patients were recruited in a naturalistic setting allowing for a large sample size, however, implying treatment with a variety of antidepressants, no standardized dosage regime, and no standardized control for plasma drug levels. Also, since $a$ priori only patients with a treatment cycle of at least 6 weeks from baseline were included in the present study, no dropouts due to non-response could be accounted for. However, there were no dropouts because of side effects. Furthermore, the sample sizes of patients with bipolar disorder, major depressive episode, and of subgroups of patients receiving a single antidepressive medication (as opposed to the more frequently used combination treatment) might have been too small to detect a minor genetic effect, thus increasing the risk of a false-negative result. Finally, although in all samples Caucasian ancestry was ascertained by Caucasian background of both parents, ethnic stratification even within the German population cannot be excluded (Steffens et al, 2006), particularly since no genomic control has been performed on the samples presently under study.

In conclusion, the present results point toward a negative influence of the higher activity COMT 158val/val genotype on antidepressant treatment response during the first 6 weeks of pharmacological treatment in major depression, possibly conferred by consecutively decreased norepinephrine and dopamine availability. The latter would be in line with a rat model of depression, where reduced dopamine availability in response to serotonergic stimulation in the nucleus accumbens, possibly conferred by the more active COMT variant, has been suggested to constitute an essential factor in the pathogenesis and course of depression as well as a target for modulation by antidepressant drugs (Zangen et al, 2001; Dremencov et al, 2004). Accordingly, given that in pharmacological studies the COMT inhibitor, tolcapone, has proven to reverse an anhedonic state in a rat model of depression and to reduce symptom severity in the treatment of major depression (Moreau et al, 1994; Fava et al, 1999), 
the present findings support a potentially beneficial effect of an antidepressive add-on therapy with substances increasing dopamine availability individually tailored according to COMT val158met genotype with homozygous carriers of the COMT 158val allele potentially profiting most. Robust identification of the COMT val158met variant as a clinical predictor for treatment response might aid in identifying the $30-40 \%$ of patients not responding to initial treatment and thus provide the basis for more efficient, economical, and time saving treatment decisions. Therefore, replication studies preferably in large, well-characterized samples are warranted to further clarify the role of COMT gene variation in antidepressant treatment response.

\section{ACKNOWLEDGEMENTS}

We gratefully acknowledge the skillful technical support of Ms Kathrin Schwarte. Contents of this paper have been presented as a sponsored poster at the Annual Meeting of the American College of Neuropsychopharmacology (ACNP), December 2006, Hollywood, FL, USA.

\section{DISCLOSURE/CONFLICT OF INTEREST}

All authors declare no conflict of interest.

\section{REFERENCES}

Alsobrook II JP, Zohar AH, Leboyer M, Chabane N, Ebstein RP, Pauls DL (2002). Association between the COMT locus and obsessive-compulsive disorder in females but not males. Am J Med Genet 114: 116-120.

Arias B, Serretti A, Lorenzi C, Gasto C, Catalan R, Fananas L (2006). Analysis of COMT gene (Val 158 Met polymorphism) in the clinical response to SSRIs in depressive patients of European origin. J Affect Disord 90: 251-256.

Barone P, Scarzella L, Marconi R, Antonini A, Morgante L, Bracco $\mathrm{F}$ et al (2006). Pramipexole $v s$ sertraline in the treatment of depression in Parkinson's disease: a national multicenter parallel-group randomized study. J Neurol 253: 601-607.

Boudikova B, Szumlanski C, Maidak B, Weinshilboum R (1990). Human liver catechol-O-methyltransferase pharmacogenetics. Clin Pharmacol Ther 48: 381-389.

Bray NJ, Buckland PR, Williams NM, Williams HJ, Norton N, Owen MJ et al (2003). A haplotype implicated in schizophrenia susceptibility is associated with reduced COMT expression in human brain. Am J Hum Genet 73: 152-161.

Cassano P, Lattanzi L, Fava M, Navari S, Battistini G, Abelli M et al (2005). Ropinirole in treatment-resistant depression: a 16-week pilot study. Can J Psychiatry 50: 357-360.

Cassano P, Lattanzi L, Soldani F, Navari S, Battistini G, Gemignani A et al (2004). Pramipexole in treatment-resistant depression: an extended follow-up. Depress Anxiety 20: 131-138.

Chen J, Lipska BK, Halim N, Ma QD, Matsumoto M, Melhem S et al (2004). Functional analysis of genetic variation in catechol-Omethyltransferase (COMT): effects on mRNA, protein, and enzyme activity in postmortem human brain. Am J Hum Genet 75: 807-821.

Cusin C, Serretti A, Lattuada E, Lilli R, Lorenzi C, Smeraldi E (2002). Association study of MAO-A, COMT, 5-HT2A, DRD2, and DRD4 polymorphisms with illness time course in mood disorders. Am J Med Genet 114: 380-390.

Dailly E, Chenu F, Renard CE, Bourin M (2004). Dopamine, depression and antidepressants. Fundam Clin Pharmacol 18: 601-607.
Davidson JR, McLeod MN, Turnbull CD, White HL, Feuer EJ (1979). Catechol-O-methyltransferase activity and classification of depression. Biol Psychiatry 14: 937-942.

Domschke K, Freitag CM, Kuhlenbaumer G, Schirmacher A, Sand $\mathrm{P}$, Nyhuis $\mathrm{P}$ et al (2004). Association of the functional V158M catechol-O-methyl-transferase polymorphism with panic disorder in women. Int J Neuropsychopharmacol 7: 183-188.

Dremencov E, Gispan-Herman I, Rosenstein M, Mendelman A, Overstreet DH, Zohar J et al (2004). The serotonin-dopamine interaction is critical for fast-onset action of antidepressant treatment: in vivo studies in an animal model of depression. Prog Neuropsychopharmacol Biol Psychiatry 28: 141-147.

Fava M, Rosenbaum JF, Kolsky AR, Alpert JE, Nierenberg AA, Spillmann $M$ et al (1999). Open study of the catechol-Omethyltransferase inhibitor tolcapone in major depressive disorder. J Clin Psychopharmacol 19: 329-335.

Frisch A, Postilnick D, Rockah R, Michaelovsky E, Postilnick S, Birman $\mathrm{E}$ et al (1999). Association of unipolar major depressive disorder with genes of the serotonergic and dopaminergic pathways. Mol Psychiatry 4: 389-392.

Funke B, Malhotra AK, Finn CT, Plocik AM, Lake SL, Lencz T et al (2005). COMT genetic variation confers risk for psychotic and affective disorders: a case control study. Behav Brain Funct 1: 19.

Kremer I, Pinto M, Murad I, Muhaheed M, Bannoura I, Muller DJ et al (2003). Family-based and case-control study of catechol-Omethyltransferase in schizophrenia among Palestinian Arabs. Am J Med Genet (Neuropsychiatr Genet) 119: 35-39.

Kunugi H, Vallada HP, Hoda F, Kirov G, Gill M, Aitchison KJ et al (1997). No evidence for an association of affective disorders with high- or low-activity allele of catechol-o-methyltransferase gene. Biol Psychiatry 42: 282-285.

Lachman HM, Morrow B, Shprintzen R, Veit S, Parsia SS, Faedda G et al (1996). Association of codon 108/158 catechol-O-methyltransferase gene polymorphism with the psychiatric manifestations of velo-cardio-facial syndrome. Am J Med Genet 67: 468-472.

Lambert G, Johansson M, Agren H, Friberg P (2000). Reduced brain norepinephrine and dopamine release in treatmentrefractory depressive illness: evidence in support of the catecholamine hypothesis of mood disorders. Arch Gen Psychiatry 57: 787-793.

Mannisto PT, Kaakkola S (1999). Catechol-O-methyltransferase (COMT): biochemistry, molecular biology, pharmacology, and clinical efficacy of the new selective COMT inhibitors. Pharmacol Rev 51: 593-628.

Massat I, Souery D, Del-Favero J, Nothen M, Blackwood D, Muir W et al (2005). Association between COMT (Val158Met) functional polymorphism and early onset in patients with major depressive disorder in a European multicenter genetic association study. Mol Psychiatry 10: 598-605.

Mattay VS, Goldberg TE, Fera F, Hariri AR, Tessitore A, Egan MF et al (2003). Catechol $O$-methyltransferase val158-met genotype and individual variation in the brain response to amphetamine. Proc Natl Acad Sci USA 100: 6186-6191.

Moreau JL, Borgulya J, Jenck F, Martin JR (1994). Tolcapone: a potential new antidepressant detected in a novel animal model of depression. Behav Pharmacol 5: 344-350.

Ohara K, Nagai M, Suzuki Y, Ohara K (1998). Low activity allele of catechol-o-methyltransferase gene and Japanese unipolar depression. Neuroreport 9: 1305-1308.

Radloff LS (1977). The CES-D scale: a self-report depression scale for research in the general population. Appl Psychol Measur 1: $385-401$.

Rush AJ, Kraemer HC, Sackeim HA, Fava M, Trivedi MH, Frank E et al (2006). Report by the ACNP Task Force on response and remission in Major Depressive Disorder. Neuropsychopharmacology 31: 1841-1853. 
Sackeim HA (2001). The definition and meaning of treatmentresistant depression. J Clin Psychiatry 62: 10-17.

Sasieni PD (1997). From genotypes to genes: doubling the sample size. Biometrics 53: 1253-1261.

Serretti A, Benedetti F, Zanardi R, Smeraldi E (2005). The influence of serotonin transporter promoter polymorphism (SERTPR) and other polymorphisms of the serotonin pathway on the efficacy of antidepressant treatments. Prog Neuropsychopharmacol Biol Psychiatry 29: 1074-1084.

Serretti A, Cusin C, Cristina S, Lorenzi C, Lilli R, Lattuada E et al (2003). Multicentre Italian family-based association study on tyrosine hydroxylase, catechol-O-methyl transferase and Wolfram syndrome 1 polymorphisms in mood disorders. Psychiatr Genet 13: 121-126.

Shulman R, Griffiths J, Diewold P (1978). Catechol-O-methyl transferase activity in patients with depressive illness and anxiety states. Br J Psychiatry 132: 133-138.

Steffens M, Lamina C, Illig T, Bettecken T, Vogler R, Entz P et al (2006). SNP-based analysis of genetic substructure in the German population. Hum Hered 62: 20-29.

Szegedi A, Rujescu D, Tadic A, Muller MJ, Kohnen R, Stassen HH et al (2005). The catechol-O-methyltransferase Val108/158Met polymorphism affects short-term treatment response to mirtazapine, but not to paroxetine in major depression. Pharmacogenomics J 5: 49-53.

Trivedi MH, Fava M, Wisniewski SR, Thase ME, Quitkin F, Warden $\mathrm{D}$ et al (2006). Medication augmentation after the failure of SSRIs for depression. $N$ Engl J Med 354: 1243-1252.

Wada T, Kanno M, Aoshima T, Otani K (2001). Dose-dependent augmentation effect of bromocriptine in a case with refractory depression. Prog Neuropsychopharmacol Biol Psychiatry 25: 457-462.

Willner P (2002). Dopamine and depression. In: DiChiara G (ed). Handbook of Experimental Pharmacology: Dopamine in the CNS. Springer: Berlin. pp 387-416.

Willner P, Hale AS, Argyropoulos S (2005). Dopaminergic mechanism of antidepressant action in depressed patients. J Affect Disord 86: 37-45.

Wittchen HU, Wunderlich U, Gruschwitz S, Zaudig M (1997). SKID-I, Strukturiertes Klinisches Interview für DSM-IV. Hogrefe: Göttingen.

Xie T, Ho SL, Ramsden D (1999). Characterization and implications of estrogenic down-regulation of human catechol$O$-methyltransferase gene transcription. Mol Pharmacol 56: 31-38.

Zangen A, Nakash R, Overstreet DH, Yadid G (2001). Association between depressive behavior and absence of serotonin-dopamine interaction in the nucleus accumbens. Psychopharmacology (Berlin) 155: 434-439.

Zhu G, Lipsky RH, Xu K, Ali S, Hyde T, Kleinman J et al (2004). Differential expression of human COMT alleles in brain and lymphoblasts detected by RT-coupled $5^{\prime}$ nuclease assay. Psychopharmacology (Berlin) 177: 178-184. 